\title{
Etude du Voice Onset Time (V.O.T) dans des séquences VCV produites par des patients francophones souffrant de paralysies récurrentielles
}

\author{
Camille FAUTH ${ }^{1,2}$, Béatrice VAXELAIRE ${ }^{2}$, Jean-François RODIER ${ }^{3}$, Pierre Philippe VOLKMAR ${ }^{3}$ et \\ Rudolph $\mathrm{SOCK}^{2,4}$ \\ ${ }^{1}$ Groupe Parole, LORIA CNRS UMR 7503, Nancy, France \\ ${ }^{2}$ Université de Strasbourg, Institut de Phonétique de Strasbourg - IPS \& U.R. 1339 Linguistique, \\ Langues et Parole - LilPa, E.R. Parole et \\ Cognition \\ ${ }^{3}$ Centre Paul Strauss - Strasbourg, Département de Chirurgie Oncologique, Centre Régional de Lutte \\ Contre le Cancer \\ ${ }^{4}$ Université Pavla Jozefa Safarika, Faculté des Lettres Košice, Slovaquie
}

\section{Introduction}

La présente étude propose d'analyser les caractéristiques spatio-temporelles de la voix de patients francophones présentant une immobilité laryngée suite à une opération de la glande thyroïde. Il s'agit ici d'évaluer les conséquences de l'immobilité laryngée sur le timing des articulateurs, à l'aide d'un corpus de logatomes (ou de séquences VCV).

Notre investigation, bien qu'acoustique, entend analyser, le Voice Onset Time les différents indices articulatori-acoustiques qui peuvent être observés à partir d'un signal acoustique continu, et ainsi remonter aux configurations articulatoires (Abry et al.,1985). Une attention particulière sera donc accordée aux différentes perturbations temporelles qu'une immobilité laryngée peut provoquer sur la voix des patients, grâce à l'étude de la réorganisation temporelles des paramètres retenus.

Notre étude se veut longitudinale puisque la voix des patients est enregistrée lors de différentes phases post-opératoires (post-opératoire 2, 3 et 4), ce qui devrait nous permettre de mettre au jour, non seulement les perturbations provoquées par l'immobilité laryngée, mais également les réajustements que les patients pourraient mettre en place au cours du temps et de leur rééducation orthophonique.

Rappelons qu'une immobilité laryngée a des conséquences acoustiques et aérodynamiques sur la voix des patients (voir (Hartl, Hans, Vaissière, Riquet, \& Brasnu, 2001) ou (Fauth et al., 2012) par ex.). Dans la plupart des cas, l'immobilité laryngée post-thyroïdectomie est transitoire, une amélioration de la qualité vocale est donc mesurable au cours du temps (Laccourreye, Le Clech, \& Santini, 2008). Il s'agit ici de savoir si cette amélioration de la qualité vocale est manifeste dans les aspects temporels du signal de parole.

\section{Procédure expérimentale}

\subsection{Locuteurs}

Cette étude est conduite à partir de sept patients francophones dont la voix a été enregistrée au Centre Paul Strauss de Strasbourg, puis dans le cabinet de différentes orthophonistes, soient cinq femmes (UPPBAS, UPPHEI, UPPHUB, UPPWAL et UPPWAN) et deux hommes (UPPLAT et UPPPAI). Tous ces patients présentent une immobilité laryngée unilatérale consécutive à une thyroïdectomie totale. 
Différents ORL ont pratiqué l'examen laryngé et le diagnostic, ce qui s'explique par le fait que le choix du praticien était laissé au patient. Des méthodes d'investigation laryngée différentes (miroir, laryngoscopie...) ont donc pu être utilisées en fonction du praticien retenu.

Ce groupe sera appelé UPP (Unilateral Paralysis Patients ou patient présentant une immobilité laryngée unilatérale), dans la suite de ce travail.

Les patients ont été enregistrés une fois par mois tout au long de leur rééducation orthophonique. Afin d'obtenir une cohorte comparable et ainsi de pouvoir conduire des analyses de variance (ANOVA à mesures répétées), seules trois phases post-opératoires ont pu être retenues : en post-opératoire 2 (soit quinze jours après l'intervention), en post-opératoire 3 (soit un mois après l'intervention) et en postopératoire 4 (c'est-à-dire deux mois après l'intervention). Pour certains locuteurs, la rééducation orthophonique n'était alors pas terminée.

En raison de contraintes hospitalières, les données en préopératoire n'ont pas pu être obtenues. En conséquence la voix des patients a été comparée avec celle de locuteurs contrôles (dorénavant LC), appariés en sexe et en âge avec le patient correspondant. Les locuteurs contrôles constituent alors la voix de référence.

\subsection{Corpus}

Notre corpus est constitué de douze logatomes (ou séquence VCV) insérés dans une phrase porteuse.

$$
\text { Ex : «Cet VCV ça. » }
$$

Les logatomes sont crées comme suit : si V1 est /i/ alors V2 est /a/ et vice versa. La consonne est l'une des 6 occlusives /p, t, k, b, d, g/. Ces consonnes ont été choisies car elles permettent l'alternance de séquences entièrement voisées $\mathrm{V} 1[\mathrm{~b} \mathrm{~d} \mathrm{~g}] \mathrm{V} 2$ et de séquences mixtes $\mathrm{V} 1[\mathrm{pt} \mathrm{k}] \mathrm{V} 2$, où les plis vocaux ne sont pas sollicités de façon continue.

Les douze logatomes sont donc les suivants : [abi], [adi], [agi], [api], [ati], [aki], [iba], [ida], [iga], [ipa], [ita] et [ika].

Il s'agit pour le locuteur de lire les logatomes qui lui sont présentés dix fois chacun, et de façon aléatoire.

\subsection{Mesures}

Le Voice Onset Time (VOT) a été mesuré selon Klatt (1975) soit comme l'intervalle allant du relâchement consonantique jusqu'à l'apparition de la structure formantique stable de la voyelle subséquente. Rappelons que Liberman et al., (1958) notent le VOT comme un indice majeur de la classification perceptive des occlusives voisées/non voisées.

Les durées du VOT obtenues ont également été normalisées en calculant le pourcentage pris par cet intervalle dans les différentes réalisations de la tenue consonantique entière. La tenue consonantique est considérée comme l'intervalle allant de la fin de la structure formantique stable de V1 au début de la structure formantique stable de V2. Le résultat ainsi obtenue est une valeur exprimée en pourcentage.

La durée relative du VOT a été calculée comme suit :

\section{Dwres absolue du VOTx100}

Temue congonantigue

\section{Équation $1:$ Durée relative du VOT exprimée en \%}

Il nous semble important de prendre en considération les valeurs normalisées en production de la parole afin de pouvoir évaluer correctement les effets de l'élasticité du signal temporel de la parole (Sock, 1998). 
En effet, une telle normalisation permet d'apprécier, à sa juste valeur, l'effet des variations de vitesses d'élocution sur les segments acoustiques, entre nos différents locuteurs. Nous pourrons ainsi évacuer toute variabilité de durées du VOT, liée simplement à des variations de vitesses d'élocution pour pouvoir déceler les véritables différences qui restent robustes malgré le changement de vitesse d'élocution.

\subsection{Analyses de données}

Afin de faciliter la lecture des résultats, ces derniers seront présentés sous forme de graphiques et de tableaux commentés.

Des analyses de variance (ANOVA) à mesures répétées ont été conduites pour toutes nos données (cf. infra pour plus de détails). Les valeurs présentées renvoient toujours aux moyennes calculées à partir des répétitions produites par nos locuteurs. Les écarts-types sont disponibles entre parenthèses.

\section{Hypothèses}

Etant donné qu'une immobilité laryngée entraine des modifications de la périodicité de la voix et des difficultés dans le contrôle du mouvement des plis vocaux et donc du voisement, nous formulons les hypothèses suivantes :

Les difficultés de voisement rencontrées par les patients pourraient avoir un impact sur la durée du VOT. En effet, celle-ci considérée comme un indice de vibration des plis vocaux et pouvant permettre ainsi la distinction des consonnes voisées des consonnes non voisées, serait modifiée. Traditionnellement, le VOT de Klatt (1975), allant du relâchement supraglottique de la consonne à l'apparition d'une structure vocalique formantiquement stable, est plus court pour les occlusives voisées que pour les occlusives non voisées. Pour les occlusives voisées, la transition CV implique uniquement un changement d'un état obstrué du conduit vocal à un état suffisamment dégagé de celui-ci pour permettre l'apparition d'une structure formantique clairement définie. En revanche, pour les occlusives non voisées, cette transition $\mathrm{CV}$ exige, outre le changement d'état du conduit vocal, une modification de la configuration de la glotte d'une position ouverte pour la consonne non voisée à une position fermée pour la voyelle. En conséquence, en raison de difficultés de maintien de vibrations laryngées, le VOT devrait alors être plus long pour les occlusives voisées (plus ou moins désonorisées, et produites avec difficulté par les patients) par rapport à celles produites par les locuteurs contrôles. Ainsi, la durée du VOT de Klatt (1975) des occlusives voisées des patients pourrait alors être comparable à celle mesurée pour leurs occlusives non voisées, rendant ainsi la distinction entre ces deux catégories potentiellement difficiles dans une perspective quantitative.

\section{Résultats}

\subsection{Remarques préliminaires}

Des analyses de variance (ANOVA) à trois facteurs: voisement, lieu d'articulation et phases d'enregistrement ont été conduites pour la variable VOT dans les deux contextes [a-i] et [i-a].

En contexte [a-i], nous avons observé un effet principal du voisement qui repose sur une différence significative entre les consonnes voisées et non voisées, $F(1,6)=430,27 p<0,000027$, toutes phases et tous lieux d'articulation confondus. Il y a un effet principal du lieu d'articulation, reposant sur une différence significative entre les consonnes bilabiales [ $\mathrm{p} \mathrm{b}$ ] et les quatre autres consonnes [ $\mathrm{t} d] \mathrm{et}$ [k g], $\mathrm{F}(2,12)=70,08 \mathrm{p}<10^{-6}$. Enfin, nous avons aussi constaté un effet principal de la phase, $\mathrm{F}(3,18)=216,14$ $\mathrm{p}<0,02$, qui met en lumière une différence significative entre la phase d'enregistrement post-opératoire 2 et toutes les autres phases d'enregistrement. 
En contexte [a-i], l'interaction entre les trois facteurs, voisement, lieu d'articulation et phases d'enregistrement, est statistiquement significative $\mathrm{F}(6,36)=51,41 \mathrm{p}<0,032$. L'interaction repose notamment sur le fait que quelque soit la consonne voisée considérée, les productions des locuteurs contrôles sont significativement différentes de celles des patients, lors de la phase d'enregistrement postopératoire 2. À partir de la phase d'enregistrement post-opératoire 4, les tests post-hoc indiquent que le facteur de voisement ne permet plus de distinguer les productions des locuteurs contrôles de celles des patients pour les consonnes voisées. En revanche, les consonnes non voisées des locuteurs contrôles et des patients en phases post-opératoires 2, 3 et 4 ne sont pas significativement différentes. En ce qui concerne le facteur lieu d'articulation, le test post-hoc révèle qu'en post-opératoire 2, la variable VOT ne permet pas toujours de distinguer les consonnes les unes des autres. Il n'existe alors plus de différence significative entre [d] et [g]. Enfin, signalons que le facteur lieu d'articulation pour la variable VOT indique que leurs productions sont significativement différentes en fonction de la consonne étudiée.

Nous avons observé un effet principal du voisement, $\mathrm{F}(1,6)=105,08 \mathrm{p}<0,0002$. Suite aux tests $L S D$, nous pouvons affirmer qu'il repose, comme attendu, sur une différence significative entre les consonnes voisées et les consonnes non voisées. Une différence significative a également pu être mise au jour pour le facteur lieu d'articulation, $\mathrm{F}(2,12)=77,03 \mathrm{p}<10^{-5}$. Les tests post-hoc révèlent que la durée du VOT est significativement différente en fonction du lieu d'articulation des différentes occlusives. Ainsi, la durée du VOT de $[\mathrm{p} \mathrm{b}]$ est différente de [t d], qui est elle-même différente de $[\mathrm{k} \mathrm{g}]$. Un effet principal de la phase a pu être mis au jour pour la variable VOT, en contexte [a-i] $\mathrm{F}(3,18)=188,02 \mathrm{p}<0,013$. La phase d'enregistrement post-opératoire 2 est significativement différente de toutes les autres phases d'enregistrement.

En contexte [i-a], il n'y a pas d'interaction significative entre les trois facteurs, voisement, lieu d'articulation et phases d'enregistrement $(\mathrm{p}=n s)$.

\section{Occlusives voisées}

Nous proposons dans cette section d'étudier la durée du VOT (Klatt, 1975) pour les occlusives voisées, soit $[\mathrm{b} \quad \mathrm{d}$ g]. Nous présenterons, pour chaque occlusive, les valeurs moyennes calculées à partir des productions des locuteurs contrôles et des patients. Compte tenu de l'importante variabilité dans les productions des patients, ces locuteurs seront ensuite analysés de façon individuelle, afin de déterminer si cette variabilité est le reflet de différences inter et/ou intra individuelles. Une conclusion partielle sera donnée pour les deux catégories de consonnes (voisées et non voisées) en fin de partie.

\subsection{Contexte [b]}
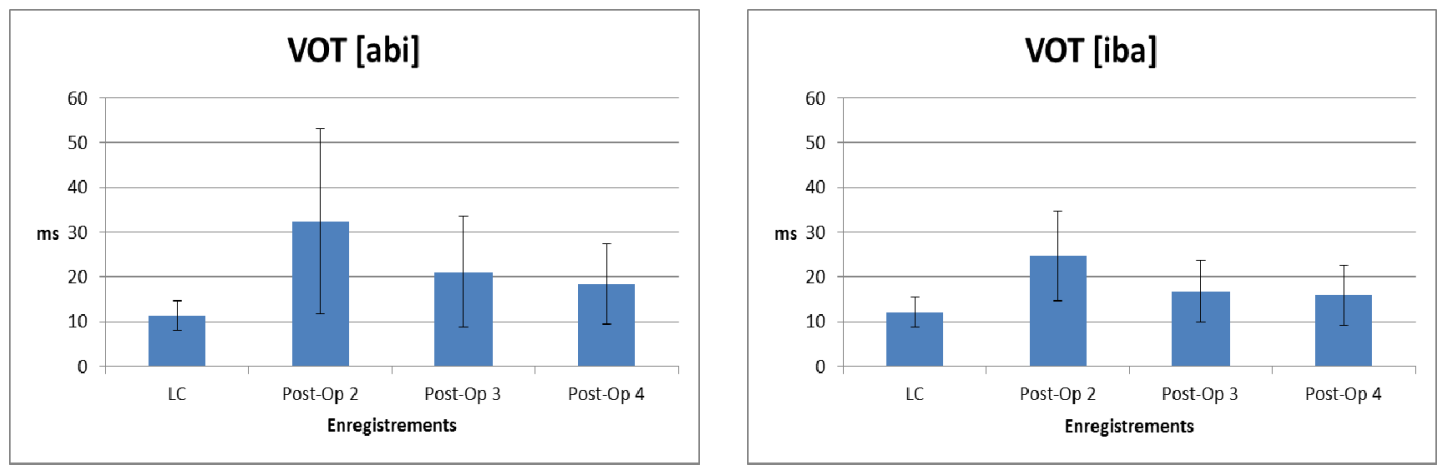

Figure 1 : Valeurs absolues de la durée du VOT (Klatt, 1975) en contexte [b] pour les logatomes [abi] (à gauche) et [iba] (à droite)

En ce qui concerne la consonne occlusive voisée [b], la durée du VOT des locuteurs contrôles est de $11,45 \mathrm{~ms}(3,34 \mathrm{~ms})$ pour [abi] et de $12,17 \mathrm{~ms}(3,39 \mathrm{~ms})$ pour [iba]; ces valeurs sont conformes à la 
littérature (cf. par ex. Sock \& Benoit, 1986) ; de plus, les écarts-types réduits témoignent d'une certaine régularité dans les productions de ces locuteurs.

En contextes [a-i] et [i-a], le VOT des patients est toujours plus long que celui des locuteurs contrôles (voir figure 1). Cette observation est particulièrement remarquable lors de la phase d'enregistrement postopératoire 2, où le VOT des patients est de $32,51 \mathrm{~ms}(20,72 \mathrm{~ms})$ pour [abi] et $24,77 \mathrm{~ms}(10,04 \mathrm{~ms})$ pour [iba]. La durée du VOT se réduit dès la phase post-opératoire 3 , où il est alors de $21,17 \mathrm{~ms}(12,41 \mathrm{~ms})$ pour [abi] et de $16,90 \mathrm{~ms}(6,88 \mathrm{~ms})$ pour [iba]. Cette tendance se poursuit jusqu'en post-opératoire 4 , où le VOT est alors mesuré à $18,48 \mathrm{~ms}(6,67 \mathrm{~ms})$ pour [abi] et à $16,01 \mathrm{~ms}(9,09 \mathrm{~ms})$.

Si dans les deux contextes, la durée du VOT est plus importante pour les patients, notamment en postopératoire 2, cela est plus notable pour le contexte [a-i] que pour le contexte [i-a]. Le VOT mesuré pour [a-i] indique également plus de variabilité que le second contexte. Nous posons que la transition [C $=>$ i] exige un contrôle plus précis et plus problématique que la transition [ $\mathrm{C}=>\mathrm{a}$ ]. En effet, la voyelle [i] requérant une constriction réduite dans la zone palatale, sans qu'il y ait de contact entre le dos de la langue et la voûte palatine, les patients semblent avoir plus de difficulté à réaliser cette transition de la consonne vers la voyelle cible [i]. Cela ne semble pas être le cas pour le [a], dont l'abaissement mandibulaire, accompagné de celui de la masse de la langue avec qui la mandibule est fortement couplée, crée sans difficulté notoire la constriction radico-pharyngale nécessaire pour l'émergence acoustique de cette voyelle.

Les phases d'enregistrement des patients sont caractérisées par des écarts-types (voir valeurs entre parenthèses) plus importants que ceux des locuteurs contrôles, ce qui peut être le résultat d'une variabilité inter et/ou intra individuelle
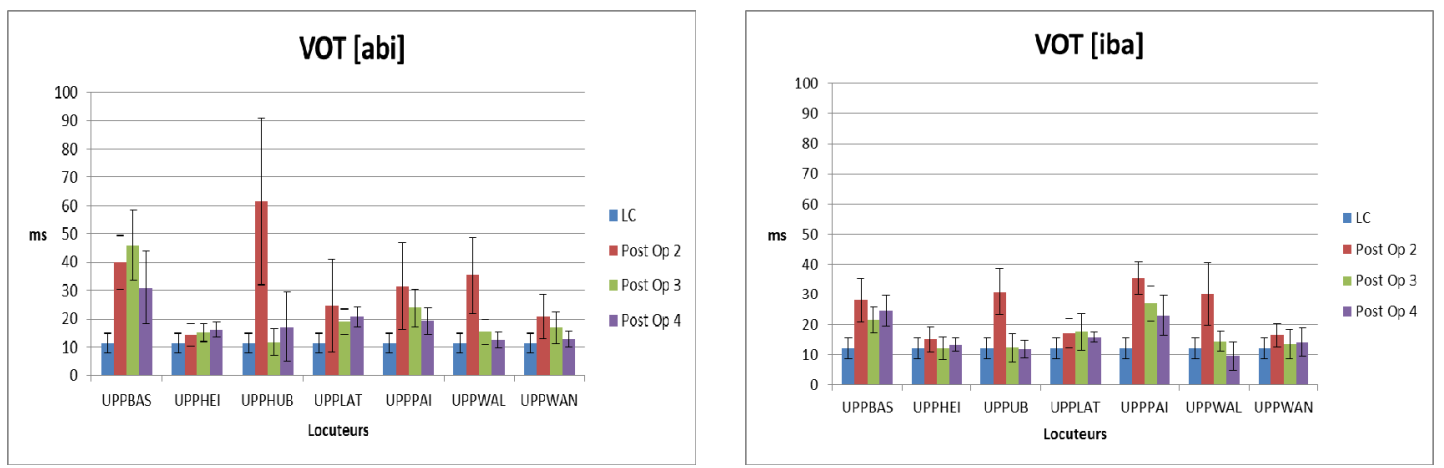

Figure 2 : Valeurs absolues de la durée du VOT (Klatt, 1975) en contexte [b] pour les logatomes [abi] (à gauche) et [iba] (à droite) en fonction du patient

La durée du VOT des locuteurs contrôles (phase en bleu) est conforme aux valeurs attendues pour la consonne [b] et est caractérisée par de faibles écarts-types, ce qui témoigne, encore une fois, de la régularité dans les productions des locuteurs contrôles.

Comme le laissaient supposer les écarts-types relevés à partir des histogrammes de la

Figure 1, si le VOT est plus long pour les patients en post-opératoire 2 que pour les locuteurs contrôles, l'importance de ce phénomène est différente en fonction du patient étudié. En effet, la durée du VOT de quatre locuteurs (de UPPBAS, de UPPHUB, de UPPPAI et de UPPWAL) est particulièrement plus élevée, alors que celle de deux locuteurs (de UPPHEI et de UPPWAL) est moins modifiée lors de cette phase d'enregistrement. Cette observation confirme le fait que la variabilité précédemment évoquée (voir firure 1) semble être en priorité inter-individuelle. Pour exemple, le VOT de UPPHUB, la patiente qui produit la durée de VOT la plus longue en post-opératoire 2 (phase en rouge), est de 31,50 ms (15,14 ms) pour [abi]. 
De plus, le contexte [a-i] (voir figure 1 à gauche) ou [i-a] (figure 1 à droite) n'a pas d'effet sur ce phénomène. Le VOT, mesuré à partir de [iba], est alors également plus long pour les patients que pour les locuteurs contrôles. Il est alors de 31,00 ms (7,69 ms) chez UPPHUB.

La phase post-opératoire 2 est également marquée par des écarts-types importants (plus de $15 \mathrm{~ms}$ en moyenne), notamment pour les productions de UPPHUB, de UPPLAT, de UPPPAI et de UPPWAL, que ce soit en contexte [a-i] ou en contexte [i-a]. Il est à noter que la variabilité en contexte [i-a] est plus réduite que celle observée en contexte [a-i]; elle reste toutefois plus importante que celle mesurée pour les locuteurs contrôles. La variabilité précédemment esquissée (voir

Figure 1) serait donc liée à des productions différentes à un double niveau: elle serait le résultat de différences inter mais également intra-individuelles.

Dès la phase d'enregistrement post-opératoire 3, la durée du VOT se réduit pour tous les locuteurs dans les deux contextes (sauf UPPBAS en contexte [a-i] pour laquelle la durée du VOT est de 45,90 ms). UPPHUB est la patiente pour laquelle la diminution de la durée du VOT est la plus importante : elle est alors de $11,80 \mathrm{~ms}(4,83 \mathrm{~ms})$ pour [abi] et $12,30 \mathrm{~ms}(4,69 \mathrm{~ms})$ pour [iba] ; rappelons que son VOT était de $61,30 \mathrm{~ms}$ pour [abi] et de $31,00 \mathrm{~ms}$ pour [iba] en post-opératoire 2. La diminution de la durée du VOT est moins importante pour les autres locuteurs. La durée du VOT est alors de 19,10 ms (4,36 ms) chez UPPPAI, par exemple. Cette phase d'enregistrement est également marquée par des écarts-types qui commencent à se réduire, ce qui indique que les productions des différents locuteurs deviennent plus régulières.

Chez UPPBAS, la durée du VOT est plus courte en post-opératoire 4 que celles mesurées lors des phases d'enregistrement précédentes, soit $31,00 \mathrm{~ms}(12,84 \mathrm{~ms})$ pour [abi] et $24,50 \mathrm{~ms}(5,02 \mathrm{~ms})$ pour [iba], sans toutefois atteindre les valeurs de référence du groupe contrôle. Rappelons que pour ce locuteur, nous n'avions pas observé de diminution du VOT, lors de la phase post-opératoire 3. Pour tous les autres locuteurs, la tendance amorcée en post-opératoire 3 se poursuit, les durées de VOT sont alors non seulement plus courtes mais également comparables à celles des locuteurs contrôles. Citons UPPWAL pour qui la durée du VOT est alors de 12,80 ms (2,70 ms), la durée du VOT du groupe contrôle étant de $11 \mathrm{~ms}(3 \mathrm{~ms})$. Cette phase d'enregistrement est également marquée par des écarts-types réduits, preuve que les productions des patients sont devenues plus régulières.

Un seul patient se démarque des observations précédentes. En effet, la durée du VOT, en contexte [a-i] ou [i-a], n'est pas modifiée en fonction des différentes phases d'enregistrement, et reste proche de celle du groupe contrôle pour la locutrice UPPHEI : elle est alors de $14,30 \mathrm{~ms}(3,86 \mathrm{~ms})$ en post-opératoire 2, de $15,20 \mathrm{~ms}(3,08 \mathrm{~ms})$ en post-opératoire 3 et de $16,10 \mathrm{~ms}(2,60 \mathrm{~ms})$ pour [abi] et de $15,10 \mathrm{~ms}(4,09 \mathrm{~ms})$ en post-opératoire 2 , de $12,10 \mathrm{~ms}(3,63 \mathrm{~ms})$ en post-opératoire 3 et de $13,30 \mathrm{~ms}(2,26 \mathrm{~ms})$ en post-opératoire 4 pour [iba]. De plus, les productions de cette locutrice sont également régulières puisque les écarts-types sont faibles, quelle que soit la phase d'enregistrement étudiée.

\subsection{Contexte $[d]$}
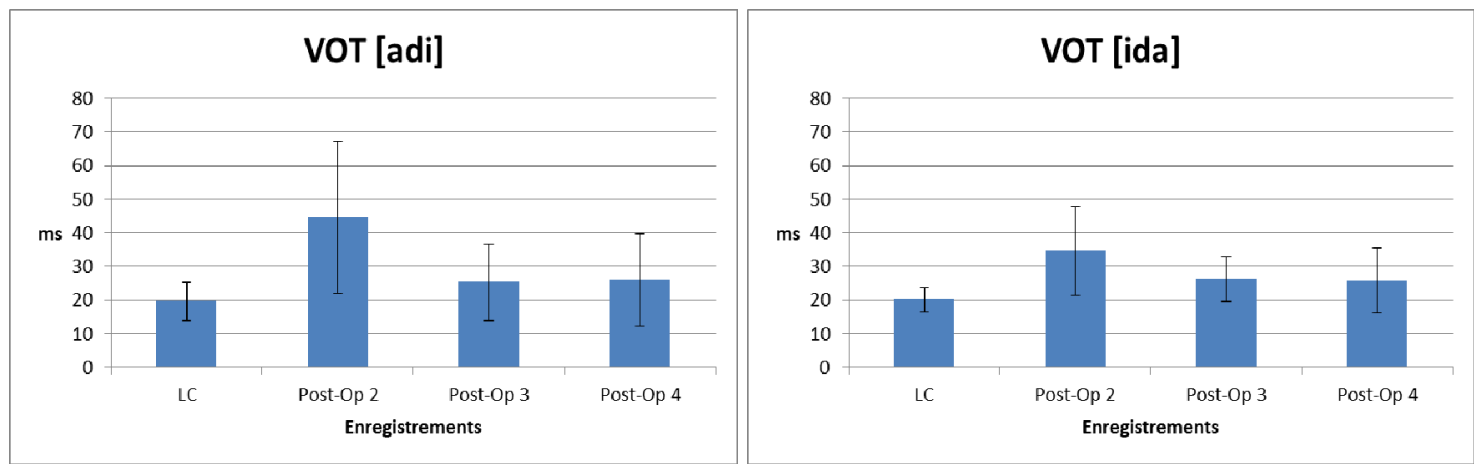
Figure 3 : Valeurs absolues de la durée du VOT (Klatt, 1975) en contexte [d] pour les logatomes [adi] (à gauche) et [ida] (à droite)

La Figure 3 illustre les valeurs de VOT pour la consonne [d]. Les durées de ce paramètre chez les locuteurs contrôles (LC) sont conformes aux valeurs attendues, à savoir 19,67 ms pour le logatome [adi] et $20,25 \mathrm{~ms}$ pour le logatome [ida]. De plus, les faibles écarts-types, soit $5,74 \mathrm{~ms}$ et 3,61 ms respectivement, témoignent de la régularité de ce groupe de locuteurs.

La première phase d'enregistrement des patients, ou la phase post-opératoire 2, est caractérisée par des durées de VOT plus longues et des écarts-types plus importants que ceux relevés chez les locuteurs contrôles. La durée du VOT est alors de 44,61 ms (22,47 ms) pour [adi] et de 34,67 ms $(13,3 \mathrm{~ms})$ pour [ida]. Ces durées diminuent dès la phase post-opératoire 3 , le VOT de [adi] étant alors $25,31 \mathrm{~ms}$ (11,54 ms) tandis que celui de [ida] est de $26,22 \mathrm{~ms}(6,63 \mathrm{~ms})$. Notons que les écarts-types se réduisent également. Cette tendance se maintient jusqu'en post-opératoire 4, où le VOT de [ida] est mesuré à 26,05 ms $(13,53 \mathrm{~ms})$ et celui de [ida] à $25,78 \mathrm{~ms}(9,64 \mathrm{~ms})$. La durée du VOT reste alors plus importante pour les patients que pour les locuteurs contrôles. Il convient toutefois de remarquer qu'elle s'est significativement réduite depuis la phase post-opératoire 2 et que les écarts-types ont diminué également, ce qui témoigne d'une amélioration du contrôle des vibrations laryngées et de la régularité des productions.
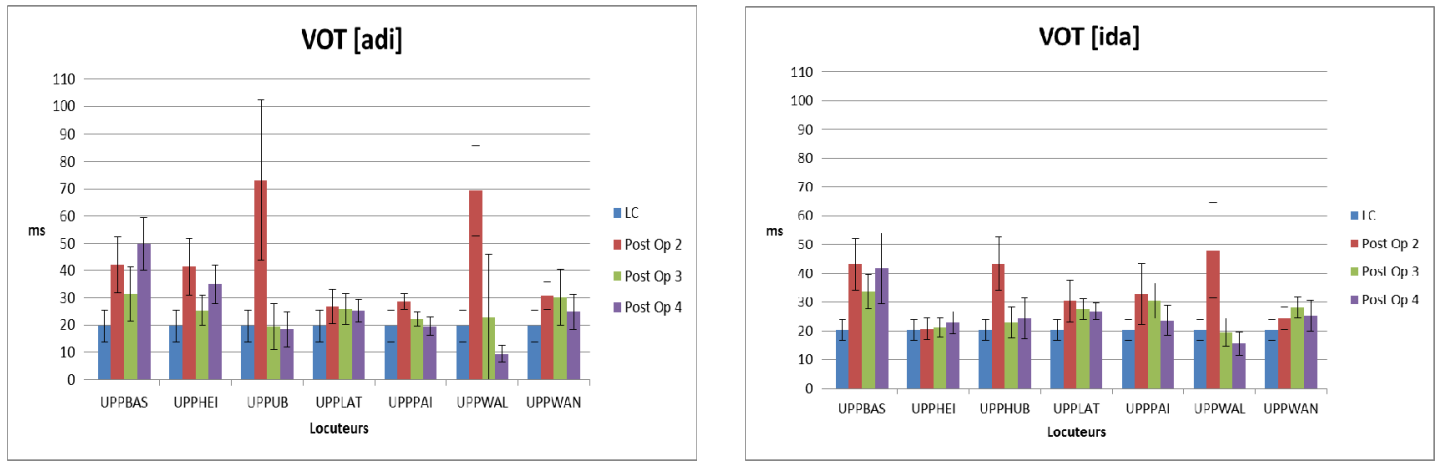

Figure 4 : Valeurs absolues de la durée du VOT (Klatt, 1975) en contexte [d] pour les logatomes [adi ](à gauche) et [ida](à droite) en fonction du patient

La Figure 4 permet d'étudier les durées de VOT pour la consonne [d] en fonction du contexte et du locuteur, afin de comprendre l'origine des écarts-types importants, relevés notamment lors de la phase d'enregistrement post-opératoire 2 (voir Figure 3). Comme attendu, les valeurs du groupe contrôle (phase en bleu) sont proches des durées moyennes attestées dans la littérature pour un locuteur sain, puisqu'elles sont à $20 \mathrm{~ms}$ et régulières, comme en témoignent les écarts-types réduits, qui s'élèvent à $5 \mathrm{~ms}$.

En ce qui concerne les productions des patients, la durée du VOT en post-opératoire 2 (phase en rouge) est toujours plus importante que celle relevée pour le groupe contrôle. L'importance de ce phénomène est toutefois à considérer en fonction du locuteur étudié. Ainsi, chez certains patients, la durée du VOT en post-opératoire 2 est beaucoup plus importante que celle relevée pour le groupe contrôle. C'est le cas notamment chez UPPBAS, UPPHUB et UPPWAL pour les deux contextes [a-i] et [i-a] ; la durée du VOT est alors de 42,20 ms $(10,30 \mathrm{~ms})$, de 73,10 ms $(29,27 \mathrm{~ms})$, de $31,50 \mathrm{~ms}(6,53 \mathrm{~ms})$, respectivement pour [adi] et de 43,10 ms $(9,16 \mathrm{~ms})$, de 43,30 ms $(9,38 \mathrm{~ms})$ et de 48,00 ms (16,51 ms). Pour ces locuteurs, la durée du VOT est multipliée au moins par deux, comparée à celle obtenue auprès des locuteurs contrôles. En ce qui concerne les autres patients (UPPHEI, UPPPAI, UPPLAT et UPPWAN), la durée du VOT est également plus importante en post-opératoire 2 que celle mesurée auprès des locuteurs contrôles, mais dans une moindre mesure. Le VOT de [adi] est alors de 41,40 ms (10,30 ms) chez UPPHEI par exemple. La même tendance est observable pour le contexte [i-a], puisque le VOT est de 20,70 ms (3,89 ms) chez la même patiente. Signalons toutefois que, pour [d], en contexte [i-a], l'augmentation de la durée du VOT en post-opératoire 2 est moins importante que celle relevée en contexte [a-i]. La phase d'enregistrement 
post-opératoire 2 est également caractérisée par des écarts-types plus importants. La variabilité précédemment évoquée (voir Figure 3) est donc le résultat de différences inter et intra individuelle, lors de cette phase d'enregistrement.

La phase d'enregistrement post-opératoire 3 (phase en vert) indique un retour vers des durées de VOT des patients comparables à celles mesurées à partir des productions des locuteurs contrôles, dans les deux contextes [a-i] et [i-a]. Une diminution de la durée de ce paramètre est donc observée pour tous les locuteurs. Il est intéressant de noter que, même les patients pour lesquels la durée du VOT était deux fois plus importante que celle relevée pour le groupe contrôle en post-opératoire 2, présentent des valeurs de VOT attendues pour un locuteur non pathologique, dès cette phase d'enregistrement. Il semble donc qu'un meilleur contrôle des vibrations laryngées est à nouveau possible pour tous les locuteurs. Citons comme exemple, le cas de UPPBAS en contexte [a-i] : la durée du VOT est de 31,30 ms (9,93 ms). La même tendance se confirme pour le contexte [i-a], où la durée du VOT est de $33,70 \mathrm{~ms}(5,91 \mathrm{~ms})$, toujours chez ce même locuteur. Quels que soient le locuteur et le contexte considérés, les écarts-types demeurent faibles.

Enfin, en post-opératoire 4 (phase en violet), les durées de VOT pour les deux contextes sont comparables à celles obtenues en post-opératoire 3, pour la plupart des locuteurs, ce qui traduit le fait que le contrôle des vibrations laryngées est maintenu. Notons que les écarts-types restent également faibles lors de cette phase d'enregistrement.

En ce qui concerne la patiente UPPBAS, les résultats indiquent une augmentation de la durée du VOT pour [adi] (VOT : 49,80 ms // écart-type : 9,80 ms) et [ida] (VOT : 59,60 ms // écart-type : 12,19 ms).

\subsection{Contexte $[g]$}

Signalons que les moyennes pour le logatome [iga] sont calculées à partir des productions de six de nos sept locuteurs. En effet, UPPPAI n'a pas été en mesure de prononcer correctement ce logatome. Ses productions ont donc dû être écartées.

Les durées de VOT pour la consonne [g] sont illustrées par la Figure 5. Les productions des locuteurs contrôles sont conformes aux valeurs attendues pour cette consonne, étant donné que l'intervalle en question mesure $30 \mathrm{~ms}$ environ, en moyenne. Les productions sont également régulières, puisque les écarts-types restent faibles, à $10 \mathrm{~ms}$. Ces observations sont valables pour les deux contextes étudiés.

Lors de la première phase d'enregistrement des patients, soit en post-opératoire 2, la durée du VOT est plus importante, à savoir $41,31 \mathrm{~ms}$ et $43,65 \mathrm{~ms}$ pour [agi] et [iga] respectivement, que celle relevée lors de la phase d'enregistrement des locuteurs contrôles. La durée du VOT pour les autres phases d'enregistrement se réduit sans toutefois atteindre les valeurs de référence des locuteurs contrôles. En contexte [a-i], la durée diminue en post-opératoire 3 , mesurée à $28,52 \mathrm{~ms}$, avant de ré-augmenter légèrement en post-opératoire 4 , où elle est mesurée à $33,25 \mathrm{~ms}$. Le contexte [i-a] suit la tendance attendue, à savoir que le VOT commence à diminuer en post-opératoire 3 , soit à $30,44 \mathrm{~ms}$, ce qui se poursuit en post-opératoire 4 , soit à $28,44 \mathrm{~ms}$.

Pour la consonne $[\mathrm{g}]$ la variabilité, remarquable grâce à des écarts-types élevés, reste importante quels que soient la phase d'enregistrement et le contexte considérés. En contexte [a-i], la variabilité perdure puisque les écarts-types sont quasiment identiques lors des trois phases d'enregistrement, soit à 17,25 ms en post-opératoire 2, à 17,50 ms en post-opératoire 3 et à $19,73 \mathrm{~ms}$ en post-opératoire 4 . En contexte [i-a], le scénario est légèrement différent, puisque la variabilité observée durant la phase d'enregistrement postopératoire 2, qui est à $20,22 \mathrm{~ms}$, diminue en post-opératoire 3, à $9,29 \mathrm{~ms}$, avant de ré-augmenter en postopératoire 4 à 14,57 ms. Ces phénomènes peuvent être attribués aussi bien à des différences inter que intra-individuelle dans les productions des patients. 

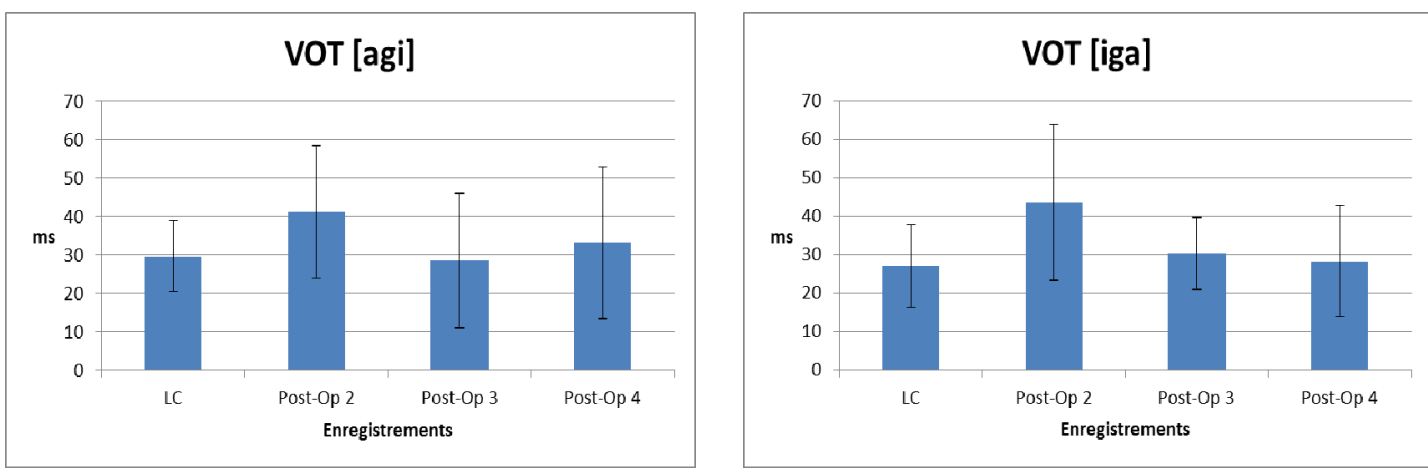

Figure 5 : Valeurs absolues de la durée du VOT (Klatt, 1975) en contexte [g] pour les logatomes [agi] (à gauche) et [iga] (à droite)

En ce qui concerne le logatome [iga], la durée du VOT est plus longue pour les patients en post-opératoire 2 que celle obtenue auprès de leurs locuteurs contrôles. Cette observation est valable pour tous les locuteurs. Le phénomène est particulièrement important pour UPPWAL, chez qui la durée du VOT est alors de $61,80 \mathrm{~ms}$, et chez UPPHUB pour qui le VOT est de 50,50 ms, en post-opératoire 2.

En contexte [i-a], la durée du VOT est plus élevée pour les patients que pour les locuteurs contrôles (toujours mesurée à $30 \mathrm{~ms}$ environ). Ce phénomène est toutefois à nuancer en fonction du locuteur étudié. En effet, si l'on considère UPPBAS (57,50 ms), UPPHUB (64,40 ms), UPPLAT (42,40 ms), UPPPAI $(30,40 \mathrm{~ms})$ et UPPWAL $(59,90 \mathrm{~ms})$, la durée du VOT est significativement plus importante en postopératoire 2 que celle de leurs locuteurs contrôles. La durée du VOT est alors au moins doublée pour ces locuteurs, comparée à celle obtenue pour leurs contrôles associés. En revanche, le VOT de UPPHEI $(24,20 \mathrm{~ms})$ et de UPPWAN (26,80 ms) n'est pas significativement plus long que celui du groupe contrôle.
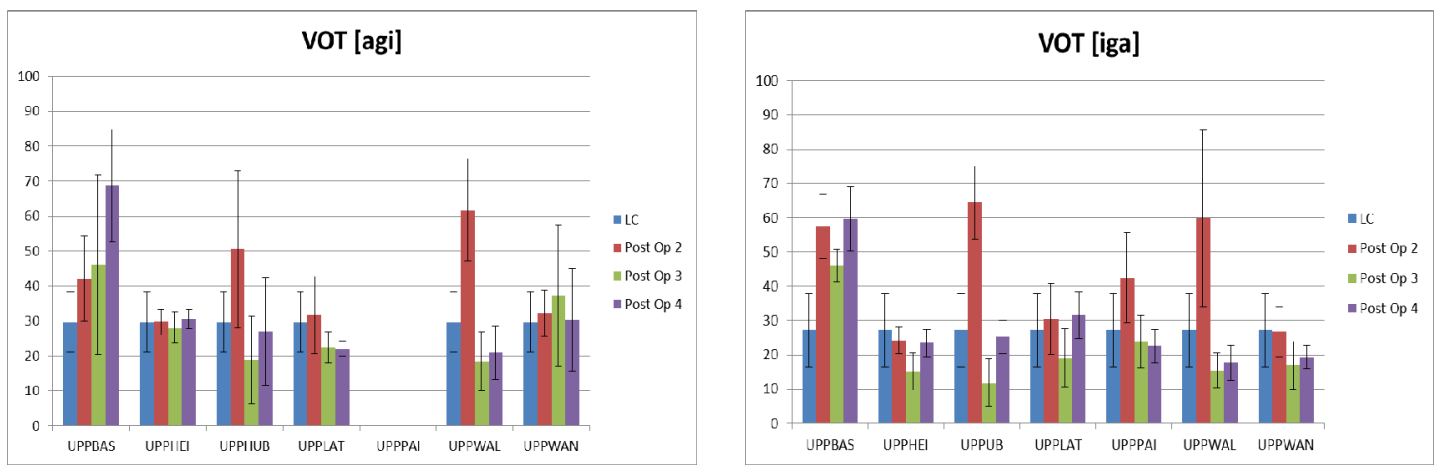

Figure 6 : Valeurs absolues de la durée du VOT (Klatt, 1975) en contexte [g] pour les logatomes [agi] (à gauche) et [iga] (à droite) en fonction du patient

En ce qui concerne la séquence [iga], la durée du VOT se réduit pour tous les patients dès la phase postopératoire 3. Elle est alors de 45,20 ms chez UPPBAS et de 24,80 ms chez UPPWAL. Quant à la phase post-opératoire 4, elle ne correspond pas au phénomène attendu. Contrairement aux autres contextes, la durée du VOT présente une forte variabilité en fonction des locuteurs étudiés pour la consonne [g]. En contexte [a-i], par exemple, la durée du VOT est plus longue que celle relevée lors des phases d'enregistrement précédentes et auprès du groupe contrôle pour UPPBAS $(68,70 \mathrm{~ms})$. Il s'agit là du VOT le plus long mesuré chez ce patient. Pour les autres locuteurs, la durée du VOT en post-opératoire 4 peut légèrement augmenter (c'est le cas pour UPPHUB : 26,90 ms), ou rester proches des mesures précédemment obtenues en post-opératoire 3 (c'est le cas pour UPPHEI : 30,50 ms). Pour ces derniers, la durée du VOT reste supérieure à celle obtenue pour les locuteurs contrôles, sans l'être statistiquement significative toutefois. Le contexte [i-a] suit le même scénario, à savoir qu'en post-opératoire 4, la durée 
du VOT est supérieure à celle mesurée en post-opératoire 3. Les mesures restent toutefois proches des valeurs de référence des locuteurs contrôles.

Il semble donc que la récupération du contrôle des vibrations laryngées soit plus problématique en contexte $[\mathrm{g}]$ que pour les deux autres occlusives voisées. Cette consonne témoigne également d'une importante variabilité quelle que soit la phase d'enregistrement étudiée. Comme attendu, la variabilité la plus importante est relevée lors de la phase d'enregistrement post-opératoire $2(22,47 \mathrm{~ms}$ chez UPPHUB pour [agi] par exemple). Elle se réduit ensuite lors des phases d'enregistrement suivantes (12,48 ms en post-opératoire 3 et 15,48 ms en post-opératoire 4 chez UPPHUB pour [agi]) mais reste plus importante que celle des locuteurs contrôles. Le contrôle du VOT serait alors plus difficile pour cette consonne rendant les productions des patients plus variables. De manière générale, nous savons (cf., par ex. Abry, et al., 1990 in Sock 1998) que le relâchement dorso-vélaire pour réaliser le [g] peut présenter plusieurs pics (trois à quatre) sur le signal acoustique, témoignant ainsi des propriétés inertielles liées à la masse importante de cette structure. Etant donné que ce relâchement ne se fait pas toujours de manière nette (comme pour celui du $[\mathrm{p}]$ ), le VOT des occlusives vélaires présente souvent une variabilité remarquable.

Nous proposons maintenant d'examiner le VOT des occlusives non voisées, avant de présenter une conclusion partielle pour les deux catégories de consonnes.

\section{Occlusives non voisées}

Nous présenterons dans cette section les résultats relatifs aux occlusives non voisées. Dans la mesure où le paramètre temporel du VOT n'a pas permis de dégager de différences significatives entre les différentes phases d'enregistrement et que la variabilité reste faible, nous ne proposerons pas d'analyse par locuteur.

\subsection{Contexte $[p]$}
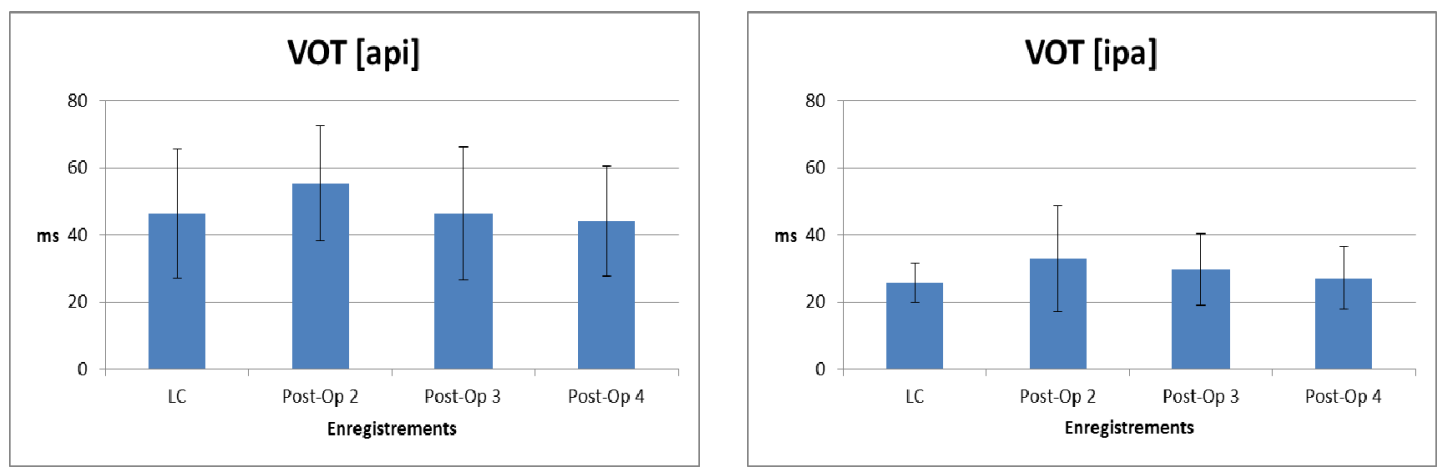

Figures 7 : Valeurs absolues de la durée du VOT (Klatt, 1975) en contexte [p] pour les logatomes [api] (à gauche) et [ipa] (à droite)

En ce qui concerne la durée du VOT pour la consonne [p], il apparait qu'elle n'est pas modifiée suite à l'immobilité laryngée. En effet, la durée de VOT des patients est plus longue (soit 55,32 ms pour [api] et $33,05 \mathrm{~ms}$ pour [ipa]) que celle mesurée à partir des productions des locuteurs contrôles (soit 46,31 ms pour [api] et $25,75 \mathrm{~ms}$ pour [ipa]), mais sans l'être significativement, que ce soit en contexte [a-i] ou en contexte [i-a]. De plus, quelle que soit la phase d'enregistrement post-opératoire considérée, les durées obtenues à partir des productions des patients restent proches de celles des locuteurs contrôles, pour les deux contextes, soit $46,27 \mathrm{~ms}$ et $44,04 \mathrm{~ms}$ pour [api], 29,71 ms et 27,10 ms pour [ipa], respectivement en phases post-opératoires 3 et 4 . 
Notons, comme constaté et expliqué précédemment, que la variabilité est plus marquée en contexte [a-i] qu'en contexte [i-a] pour toutes les phases d'enregistrement, et cela autant pour les locuteurs contrôles que pour les patients.

\subsection{Contexte [t]}
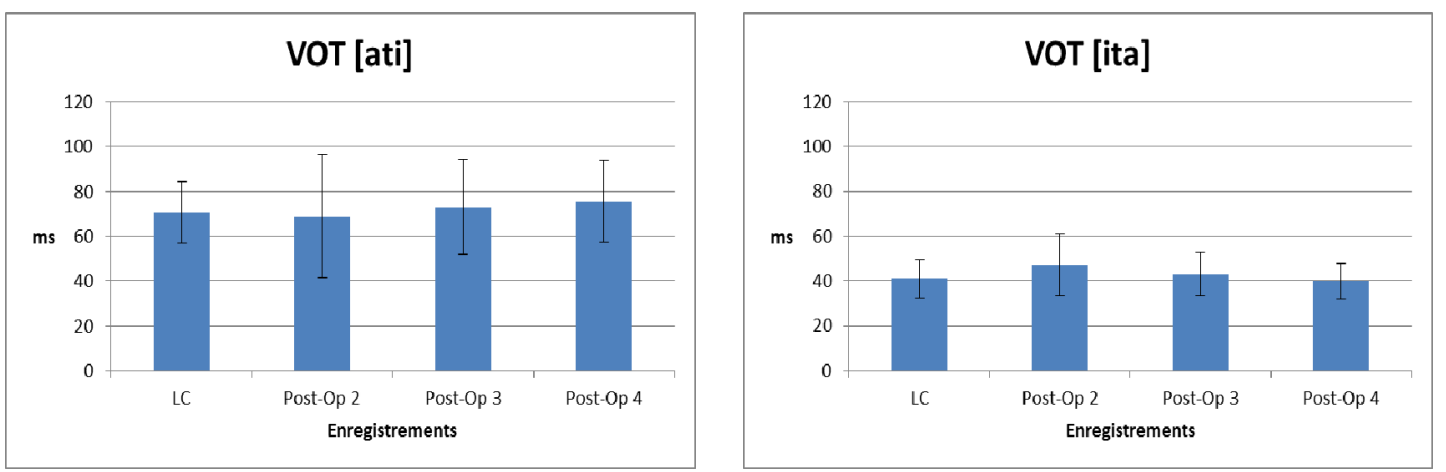

Figure 8: Valeurs absolues de la durée du VOT (Klatt, 1975) en contexte [t] pour les logatomes [ati] (à gauche) et [ita] (à droite)

Le scénario est le même pour l'occlusive [t], à savoir que la durée du VOT n'est pas modifiée par l'immobilité laryngée pour les patients par rapport aux locuteurs contrôles, même en phase d'enregistrement post-opératoire 2. Les valeurs des patients restent donc proches des valeurs de référence des locuteurs contrôles (70,75 ms pour [ati] et $41,18 \mathrm{~ms}$ pour [ita]) en contexte [a-i], soit 69,00 ms en post-opératoire $2,73,10 \mathrm{~ms}$ en post-opératoire 3 et 75,57 $\mathrm{ms}$ en post-opératoire 4 ; soit 47,21 ms en postopératoire 2, 43,24 ms en post-opératoire 3 et 40,01 ms en post-opératoire 4 .

Si l'on considère le contexte [a-i], la variabilité, traduite pas les valeurs des écarts-types, est marquée à la fois pour les locuteurs de contrôle mais aussi pour les patients, et cela dans toutes les phases d'enregistrement. Elle est la plus importante pour la phase post-opératoire 2, à 27,47 ms, puis se réduit à partir des phases post-opératoires 3 et 4 , à $21,31 \mathrm{~ms}$ et à $18,26 \mathrm{~ms}$, respectivement.

En revanche, les écarts-types sont faibles pour le contexte [i-a] : ils sont de 8,44 ms pour les productions des locuteurs contrôles, de 13,71 ms en post-opératoire 2, de 9,67 ms en post-opératoire 3 et de 7,90 ms en post-opératoire 4. De surcroît, la variabilité suit la tendance attendue, à savoir qu'elle est la plus faible pour les productions des locuteurs contrôles, la plus importante en post-opératoire 2, avant de se réduire pour les phases d'enregistrement post-opératoires 3 et 4 .

\subsection{Contexte [k]}

L'occlusive [k] répond au même schéma que les deux occlusives non voisées décrites précédemment, à savoir que les valeurs des patients restent proches des valeurs des locuteurs contrôles pour les deux contextes, et cela quelle que soit la phase d'enregistrement post-opératoire étudiée. La valeur de référence des locuteurs contrôles pour le contexte [a-i] est de $68,00 \mathrm{~ms}(11,00 \mathrm{~ms})$; en post-opératoire 2 , la durée du VOT est de 71,42 ms (22,49 ms), alors qu'elle est de 77,37 ms $(20,87 \mathrm{~ms})$ en post-opératoire 3 , et de $71,81 \mathrm{~ms}(18,98 \mathrm{~ms})$ en post-opératoire 4 . La durée de cet intervalle est donc plus importante, sans l'être significativement, pour les patients que pour celle obtenue auprès des locuteurs contrôles. La même trame est observable pour le contexte [i-a], où le VOT est de $52,08 \mathrm{~ms}(12,07 \mathrm{~ms})$ en post-opératoire 2, de 43,92 $\mathrm{ms}(9,58 \mathrm{~ms})$ en post-opératoire 3 et de $45,40 \mathrm{~ms}(10,22 \mathrm{~ms})$ en post-opératoire 4 . L'intervalle est de $45,75 \mathrm{~ms}(7,89 \mathrm{~ms})$ pour les locuteurs contrôles.

Notons, encore une fois, que la variabilité, quantifiée à l'aide des écarts-types, est plus marquée pour le contexte [a-i] que pour le contexte [i-a]. Pour [aki], comme attendu, la variabilité est plus importante lors des phases d'enregistrement des patients que pour celle des locuteurs contrôles. Signalons cependant que 
cette même variabilité se réduit au cours du temps. En revanche, pour [ika] les écarts-types sont faibles pour les deux groupes de locuteurs.
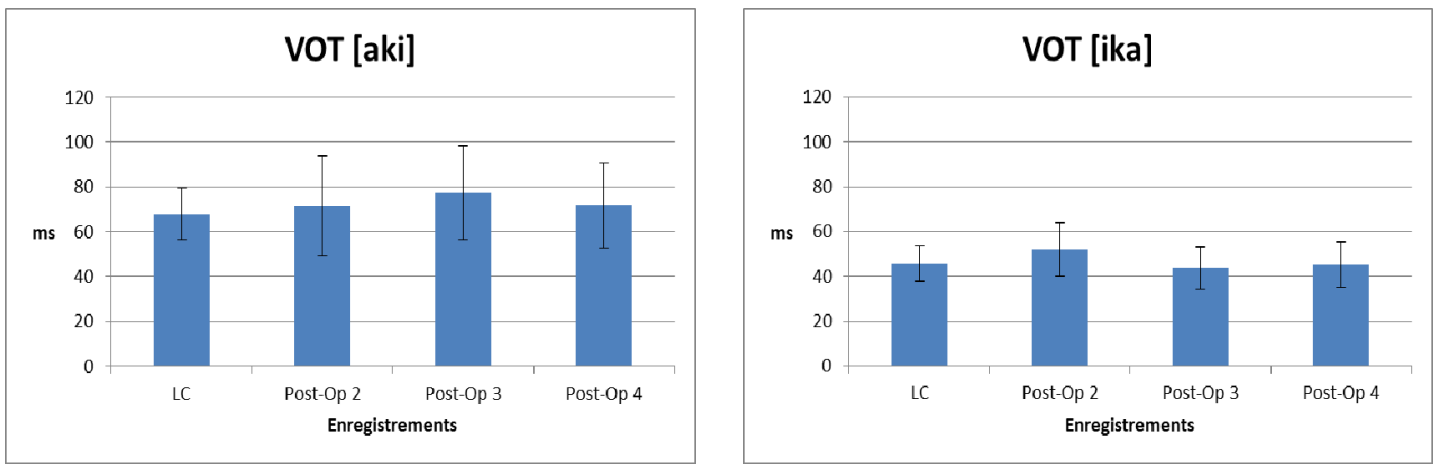

Figure 9 : Valeurs absolues de la durée du VOT (Klatt, 1975) en contexte [k] pour les logatomes [aki] (à gauche) et [ika] (à droite)

\section{Le VOT comme indice de lieu d'articulation}

Rappelons que le paramètre du VOT peut varier en fonction du lieu d'articulation. En effet, il a été observé, entre autres, que plus la closion a lieu à l'arrière de la cavité buccale, plus le VOT est long (Peterson \& Lehiste, 1962). Cela voudrait dire que plus l'aire du contact est étendue, plus le VOT est élevé (Stevens, 1989). Ainsi le VOT est généralement plus court pour les consonnes bilabiales et plus long pour le $[\mathrm{k}]$, le [t] ayant une durée intermédiaire dans la plupart des cas. Par ailleurs, on sait que le VOT de Klatt (1975) est plus long pour les occlusives non voisées que pour les occlusives voisées (Sock et Benoit, 1986). Traditionnellement en français, la durée du VOT d'un [b] est plus petite que celle du VOT d'un [d] qui est elle-même plus petite que celle d'un [g]. Le VOT est également plus court pour un [p] que pour un [t] ou un [k]. Si les données qui viennent d'être mentionnées valent pour des sujets sans trouble de la parole, la Figure 10 indique que ces constatations se maintiennent chez des locuteurs présentant une paralysie récurrentielle après thyroïdectomie, et cela pour les deux contextes étudiés, ainsi que pour toutes les phases d'enregistrement. Si la durée du VOT est effectivement allongée en postopératoire 2 pour la production des occlusives voisées des patients, celles-ci n'en restent pas moins quantitativement différenciées.
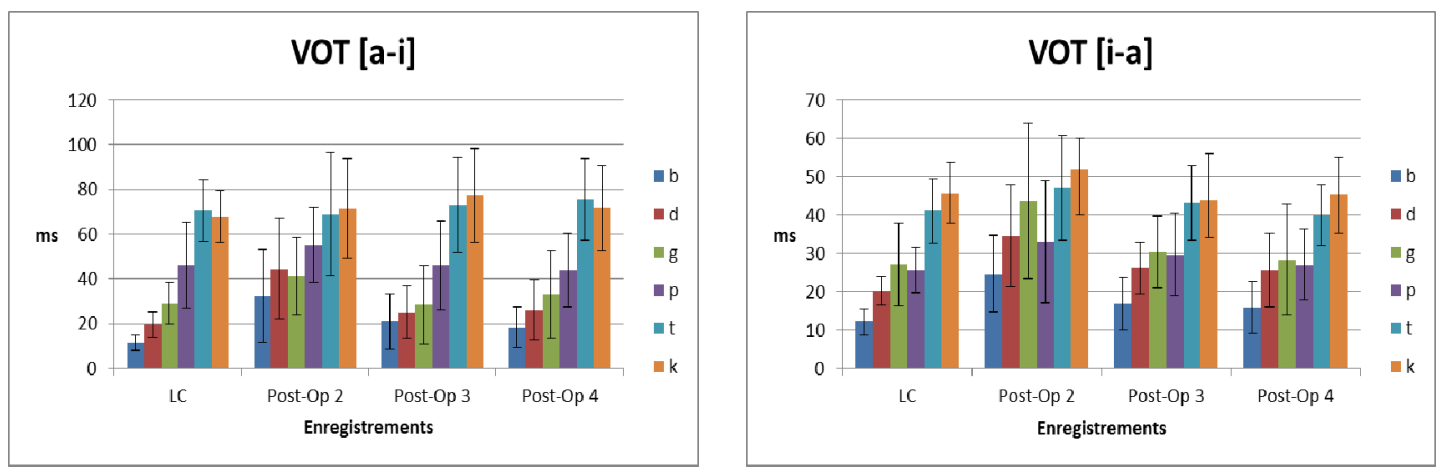

Figure 10 : Durées moyennes du VOT (Klatt, 1975) pour six occlusives du français en contexte [a-i] (à droite) et en contexte [i-a] (à gauche)

\subsection{Durées relatives du VOT}

Cette section traitera des durées relatives du VOT. Comme nous l'avons précisé plus haut, si les données temporelles en valeurs absolues permettent, certes, de quantifier l'ampleur d'un paramètre quelconque 
dans la différentiation de catégories phonétiques et/ou phonologiques, les valeurs relatives, elles, offrent in fine la possibilité d'apprécier le maintien et la robustesse ou non de ce paramètre dans des conditions de réalisation différentes. Sur le plan temporel, ce sont essentiellement les différences de vitesses d'élocution intrinsèques aux locuteurs qui sont les sources majeures de l'élasticité du signal de la parole (Gaitenby, 1965 ; Sock, 1998).

La figure 11 indique que les observations faites pour les valeurs absolues sont confirmées lors de l'étude des valeurs relatives. Les durées relatives du VOT des consonnes voisées restent plus longues pour les patients présentant une immobilité laryngée que celles des locuteurs contrôles. Cela est particulièrement remarquable en contexte [a-i] (à gauche sur la figure 11). Les durées les plus importantes sont alors relevées en post-opératoire 2 (en rouge sur la figure 11), puisque le VOT a été quantifié à 22,93\% $(10,15 \%)$ pour [b], à $31,61 \%(9,21 \%)$ pour [d] et à $26,99 \%(6,96 \%)$ pour [g]. Les durées se réduisent ensuite en post-opératoire 3 , les occlusives voisées étant alors mesurées à $17,07 \%(8,55 \%), 21,35 \%$ $(7,68 \%), 20,49 \%(8,69 \%)$ respectivement. Ces valeurs se maintiennent jusqu'en post-opératoire 4 , soit $15,36 \%(6,67 \%)$ pour [b], $21,10 \%(7,44 \%)$ pour [d] et $22,88 \%(8,28 \%)$ pour [g]. Notons que cette dernière phase est également caractérisée par une variabilité réduite.

Les mêmes observations, dans une moindre mesure toutefois $(\mathrm{p}=\mathrm{ns})$, sont observables en contexte [i-a], à droite sur la figure 11. Ces constats étaient notés en valeurs absolues également. Il est donc probable que la transition $\left[\mathrm{C}_{\text {voisée }}=>\right.$ i] exige un contrôle plus précis et plus problématique que la transition $\left[\mathrm{C}_{\text {voisée }}=>\right.$ a]. La durée relative du VOT de [b d g] est plus importante lors de la phase post-opératoire 2 (en rouge sur la figure 11), étant donné que le VOT de [b] était quantifié à 20,38\% (8,65\%), celui de [d] à 29,08\% $(8,14 \%)$ et celui de $[\mathrm{g}]$ à $32,96 \%(10,41 \%)$ à cette date. Dès la phase d'enregistrement suivante (en vert sur la figure 11), les durées diminuent pour les trois consonnes : le [b] est à $16,75 \%(8,33 \%)$, le [d] à $26,80 \%(7,30 \%)$ et le [g] à 29,95 (8,51\%). Ces valeurs se maintiennent en post-opératoire 4 (en violet sur figure 11$): 14,82 \%(6,89 \%)$ pour [b], 25,17\% (7,66\%) pour [d] et $26,29 \%(9,81 \%)$ pour [g].

Les durées relatives du VOT pour les consonnes non voisées ne permettent pas de distinguer les différentes phases d'enregistrement $(\mathrm{p}=\mathrm{ns})$, et ce dans les deux contextes [a-i] et [i-a].

Les écarts-types les plus importants sont relevés lors de la phase d'enregistrement post-opératoire 2, la variabilité se réduisant dans les phases d'enregistrement suivantes.
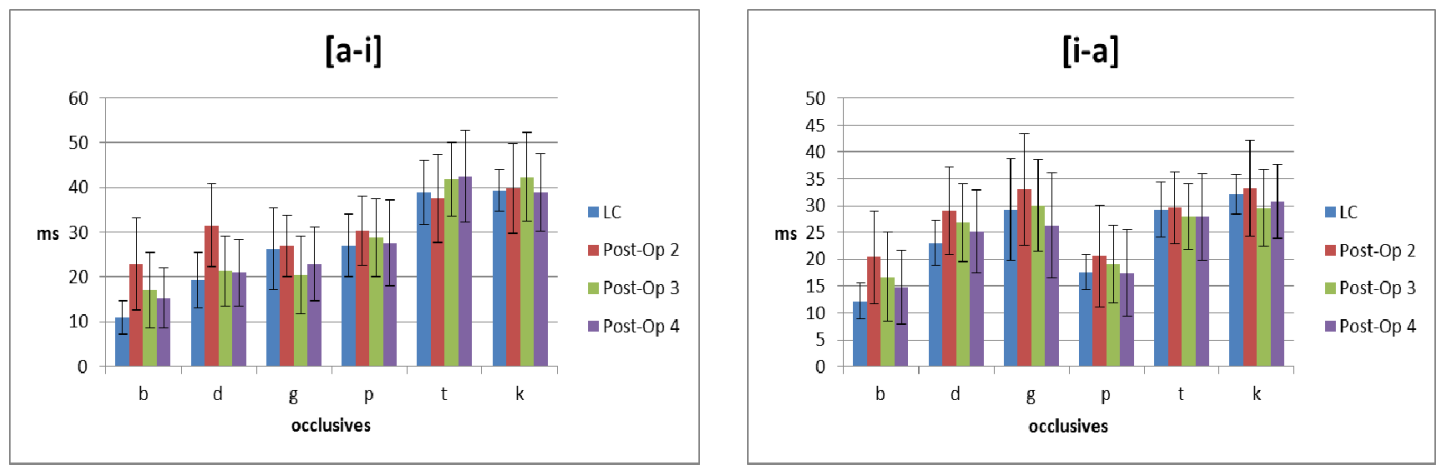

Figure 11: Durées relatives moyennes du VOT (Klatt, 1975) pour six occlusives du français en contexte [a-i] (à droite) et en contexte [i-a] (à gauche)

\section{Discussion}

Il convient à présent de vérifier si nos hypothèses initiales ont été confirmées ou infirmées, en ce qui concerne le timing des paramètres retenus. 
Les difficultés de voisement rencontrées par les patients ont un impact sur la durée du VOT (Hypothèse 1). En raison de difficultés de maintien de vibrations laryngées, le VOT est plus long pour les occlusives voisées produites par les patients que celles produites par les locuteurs contrôles. En effet, les vibrations périodiques s'estompent plus tôt, «laissant ainsi la place », de manière précoce, au relâchement consonantique. Cela est particulièrement vrai lors de la phase d'enregistrement post-opératoire 2. La durée du VOT de Klatt (1975) des occlusives voisées des patients reste quantitativement différentes de celle mesurée pour leurs occlusives non voisées; la distinction entre ces deux catégories devrait rester possible dans une perspective perceptive. Signalons que dans certains cas, les occlusives voisées, produites par les patients dans les phases d'enregistrement post-opératoires précoces, sont plus ou moins désonorisées puisque produites avec une certaine difficulté de maintien de l'activité laryngienne.

Conformément à la deuxième hypothèse, les différences de durées observées en valeurs absolues dans l'hypothèse 1 sont maintenues en valeurs relatives. Rappelons que le VOT est plus long en postopératoire 2 pour les occlusives voisées des patients. Il semble donc que l'allongement du VOT pour les occlusives voisées dans les productions des patients en post-opératoire 2 soit une manœuvre robuste. Cette différence de VOT ne proviendrait pas alors d'une variation de la vitesse d'élocution.

Notons que c'est dans le contexte [a-i] que se manifestent les plus grandes différences entre les productions des patients et celles des locuteurs contrôles. Il est donc probable que la transition $[\mathrm{C}=>\mathrm{i}]$ exige un contrôle plus précis et plus problématique que la transition [C=> a]. En effet, la voyelle [i] requérant une constriction réduite dans la zone palatale, sans qu'il y ait de contact entre le dos de la langue et la voûte palatine, les patients semblent alors avoir plus de difficultés à réaliser cette transition de la consonne vers la voyelle cible [i] de petite aperture dite «fermée ». Cela ne semble pas être le cas pour le [a], dont l'abaissement mandibulaire, avec translation vers l'arrière, accompagné de celui de la masse de la langue avec qui la mandibule est fortement couplée, crée sans difficulté la constriction radicopharyngale nécessaire pour l'émergence de l'identité acoustique de cette voyelle.

Nos données indiquent que la variabilité, qu'elle soit intra ou interindividuelle, diminue à partir de la phase post-opératoire 3, révélant ainsi une certaine stabilisation du timing des paramètres temporels retenus. Avec le temps et la rééducation, l'on observe non seulement une réduction de la variabilité signalée ci-dessus, mais également des valeurs temporelles qui ressemblent de plus en plus à celles des locuteurs contrôles.

\section{Conclusions}

Dans cette étude, nous avons étudié les modifications de la durée du V.O.T pour sept patients francophones présentant une immobilité laryngée, suite à une opération de la glande thyroïde. Notre étude se voulait longitudinale, puisque les patients ont été enregistrés à plusieurs reprises (en post-opératoire 2, 3 et 4) lors de leur rééducation orthophonique.

Seul l'intervalle du VOT pour les occlusives sonores a été modifié. Pour les occlusives voisées, la transition CV implique uniquement un changement d'un état obstrué de conduit vocal à un état suffisamment dégagé de celui-ci pour permettre l'apparition d'une structure formantique claire et stable. En revanche, pour les occlusives non voisées, cette transition CV exige, outre le changement d'état du conduit vocal, une modification de la configuration de la glotte d'une position ouverte pour la consonne non voisée à une position fermée pour la voyelle subséquente. En conséquence, en raison de difficultés de maintien de vibrations laryngées, le VOT, qui est un indice de voisement, est alors généralement plus long pour les occlusives voisées que celles produites par les locuteurs contrôles. La durée du VOT de Klatt (1975) des occlusives voisées des patients reste toutefois quantitativement distincte des occlusives non voisées. En conséquence, la durée de la tenue consonantique des patients se voit également allongée dans les phases d'enregistrement précoces.

D'autres intervalles spatio-temporels acoustiques comme le Voice Termination Time (Agnello, 1975) ou délai d'arrêt du voisement, la durée de l'occlusion (Meynadier \& Gaydina, 2012) ou la tenue consonantique et vocalique devraient également nous permettre de mieux appréhender de façon plus 
globale les stratégies de réajustements que les patients mettent en place suite à l'apparition d'une immobilité laryngée. Enfin, d'autres paramètres articulatoires mériteraient d'être explorés afin de compléter les résultats présentés ici, et d'apporter davantage de robustesse et de précisions aux interprétations proposées dans ce travail. Cette démarche devrait permettre de consolider nos résultats à l'aide de modèles géométriques du conduit vocal et de modèles biomécaniques (Laprie \& Busset, 2011 ; (Perrier, et $a l$. 2011). Il serait utile aussi de procéder à une vérification de la validité perceptive de ces réorganisations spatio-temporelles en conduisant des tests de perception.

\section{Remerciements}

Programme de la MISHA, 2013-2017, ANR "DOCVACIM" et le projet du CS de UdS GutenbergStrasbourg, 2009-2011

\section{Références bibliographiques}

Abry, C., Orliaguet, J.-P., \& Sock, R. (1990). Patterns of speech phasing. Their robustness in the production of a timed linguistic task: single vs. double (abutted) consonants in French. Cahiers de psychologie cognitive, 10(3), 269- 288.

Fauth, C., Vaxelaire, B., Rodier, J., Volkmar, P., Bouarourou, F., Hirsch, F., \& Sock, R. (2012). Etude acoustique de voyelles soutenues produites par des patients opérés de la thyroïde souffrant ou non de paralysies récurrentielles. In Actes de la conférence conjointe JEP-TALNRECITAL 2012 (Vol. 1, p. 205 - 112). Grenoble.

Gaitenby, J. (1965). The elastic word. Speech Research, 2, 1- 12.

Hartl, D., Hans, S., Vaissière, J., Riquet, M., \& Brasnu, D. (2001). Objective Voice Quality Analysis Before and After Onset of Unilateral Vocal Fold Paralysis. Journal of Voice, 15(3), $351-361$.

Klatt, D. (1975). Voice Onset Time, Frication, and Aspiration in Word-Initial Consonant Clusters. J Speech Hear Res, 18(4), 686- 706.

Laccourreye, O., Le Clech, G., \& Santini, J. (2008). Complications de la chirurgie des affections et tumeurs bénignes de la thyroïde. In Les risques chirurgicaux en oto-rhino-laryngologie : information, prise en charge et prévention (Société francaise d'oto-rhino-laryngologie., p. 369- 386). Paris: Laccourreye O, Chabolle F.

Laprie, Y., \& Busset, J. (2011). Construction and evaluation of an articulatory model of the vocal tract. Présenté à 19th European Signal Processing Conference - EUSIPCO-2011.

Liberman, A. M., Delattre, P. C., \& Cooper, F. S. (1958). Some cues for the distinction between voiced and voiceless stops in initial position. Language and Speech, 1, 153_167.

Perrier, P., Payan, Y., Buchaillard, Stéphanie, I., Nazari, M. A., \& Chabanas, M. (2011). Biomechanical models to study speech. Faits de Langues, 37, 155-171.

Peterson, G., \& Lehiste, I. (1962). Duration of syllable Nuclei in American English. Journal of the Acoustical Society of America, (32), 693- 703.

Sock, R. (1998). Organisation temporelle en production de la parole: émergence de catégories sensori-motrices phonétiques. Presses univ. du Septentrion, Université Stendhal, Grenoble III.

Sock, R., \& Benoit, C. (1986). VOT et VTT en français. In 15 èmes Journées d'Etude sur la Parole du Groupe Communication Parlée (p. 307- 310). Aix-en-Provence.

Stevens, K. (1989). On the quantal nature of speech. Journal of Phonetics, (17), 3- 45. 